This is the final peer-reviewed accepted manuscript of:

Prandi, C., Roccetti, M., Salomoni, P. et al. Fighting exclusion: a multimedia mobile app with zombies and maps as a medium for civic engagement and design. Multimed Tools Appl 76, 4951-4979 (2017).

The final published version is available online at: https://doi.org/10.1007/s11042$\underline{016-3780-9}$

Rights / License:

The terms and conditions for the reuse of this version of the manuscript are specified in the publishing policy. For all terms of use and more information see the publisher's website. 


\title{
Fighting exclusion: a multimedia mobile app with zombies and maps as a medium for civic engagement and design
}

\author{
Catia Prandi ${ }^{*}$, Marco Roccetti ${ }^{1}$, Paola Salomoni ${ }^{1}$, Valentina Nisi ${ }^{2}$, Nuno Jardim Nunes ${ }^{2}$ \\ ${ }^{1}$ Department of Computer Science and Engineering, \\ University of Bologna, \\ Mura Anteo Zamboni 7, 40126, Bologna, Italy \\ ${ }^{2}$ Madeira Interactive Technologies Institute, \\ University of Madeira, \\ Caminho da Penteada, 9020-105, Funchal, Portugal \\ *Corresponding author: E-mail: catia.prandi2@,unibo.it \\ Tel: +39-0547-338813 \\ Fax: +39-0547-338890
}

\begin{abstract}
This paper presents a study on urban data crowdsourcing driven by Geo-Zombie, a multimedia mobile application we designed and developed to engage pedestrians in taking note of urban architectural impediments and facilities by documenting them through pictures and multimedia data. Geo-Zombie aims at transforming the civic activity of contributing into a virtual gamified experience where players attempt to escape from horrific situations in which zombies are ready to cannibalize unsuspecting walkers. In some sense, walkers that kill zombies deeply reconnect with the concept of imminent danger which can be fought resorting to appropriate civic actions. To challenge our initial hypotheses we conducted a design process, starting with a concept generation where three different concepts were discussed which gave rise to five different multimedia mobile apps including the one with zombies. Then, focus group, experience prototyping, application design and implementation, and finally field trials were exploited to refine the design and to select the best apps out of the five that better responded to the need of involving common people in collecting urban accessibility data. It is worth noting that the experiences of use with 50 avid walkers have demonstrated that a multimedia mobile app with maps and zombies can be a concrete step towards a social inclusion strategy while inviting new reflections and discussions on the issue of urban data crowdsourcing.
\end{abstract}

Keywords: urban accessibility, crowdsourcing, social inclusion, gamification, multimedia mobile app 


\section{Introduction}

Since 1968, with the debut of the Romero's movie Night of the Living Dead [46], until the present days with AMC's The Walking Dead, one of the highest rated shows in the cable television history [10], zombies have raised our attention on how humans "react or fail to react, or react stupidly" to societal apocalyptic events [47]. In the decades since, according to Romero's vision, zombies have become a privileged tool of interpretation of our contemporary society, as their analysis poses many fundamental questions regarding the human condition and people's overall concerns and fears about modern consumerism [39]. With this in view, an interesting issue emerged in the zombies film literature: the horrific nature of the undead creatures and humans' behavior sadly intersect, as humans often behave selfishly, ready to turn on one another and unable to help others [39]. "The humans are the ones I dislike the most, and they're where the trouble really lies" emphasizes G. A. Romero [48], revealing one of the lessons that the zombies metaphor seems to teach us: on the eve of an apocalypse the only behaviors that can save mankind are those based on collaboration. Collaboration and cooperation are posed as the only acts of cultural and civic engagement that may preserve our community [20]. Social recruiting proliferates hence in the zombie genre as a unique medium to defend an impoverished world from the zombie apocalypse [29].

Setting the zombie phenomenon apart for a while, in recent years we have seen the emergence of a myriad of software applications exploiting geographical maps and spatial representations to provide support to the different driving, biking, running and walking activities. These applications typically leverage on Geographical Information Systems (GIS) data and algorithmically produce paths and routes that humans avidly consume, with the presupposition that those maps will lead them to their destination, through a planned and precise sequence of steps, usually in the shortest possible time. Unfortunately, the aim for efficiency, speed and control, which is at the basis of the use of all this computational cartography, does not always fit squarely with the physical condition of a vast portion of people characterized by special needs and different abilities. In their attempt to find the shortest route to a given destination, in fact, these automatic procedures often lead people with inabilities to follow a given disconnected path, therefore determining a modern and algorithmic form of social segregation. Think of Google Maps [19], for example. This service gives route information for both cars and pedestrians, but fails to provide any indication concerning the accessibility of the paths it suggests, as no digital data refers to this aspect, 
thus impeding to algorithms to consider accessibility for people with impairments [34]. Engaging somebody to take note of architectural impediments by documenting through pictures a number of non-accessible curbs, as well as recording blind audible traffic signals of as many intersections as possible, would be a first move towards reconciling people with disabilities with the algorithmic world they inhabit (see e.g. $[22,42,45])$.

Drawing on studies on the role of playful elements placed at the service of our society and culture (see e.g. $[13,32,45]$ and algorithmic living (see e.g. $[12,31,49]$ ), we designed and developed a multimedia GIS-enabled social tool for engaging pedestrians in documenting architectural barriers, called Geo-Zombie. Geo-Zombie is a multimedia mobile application that transforms the civic activity of mapping urban impediments into the attempt to escape from horrible situations where zombies are ready to cannibalize unsuspecting walkers. Through their smart phones, in fact, walkers label the surrounding environment signaling the presence of architectural barriers. Then, these data, integrated with the GPS coordinates of their smart phones, are sent to a remote server that continuously updates a digital map with city accessibility information. As soon as walkers report on a given architectural barrier (or a facility), game points are acquired and used to fire super-bouncy bullets that eliminate all zombies that suddenly appear while using the app. Granted as a reward to walkers, ammunitions to contrast zombies take on an apotropaic value. Walkers who kill zombies, in fact, deeply connect with the concept of imminent danger, which can be fought resorting to appropriate civic actions. Reporting on urban impediments here becomes the way walkers protect against evil and deflect its harmful influences. The human attitude of resignation in the face of the many natural impediments with which the world is filled is then overwhelmed by the simplicity through which zombies are supposed to be eliminated. Thereby, fighting zombies becomes an allegory that represents the efforts human beings should undertake to alleviate the conditions of those who have been relegated to the fringe of our society.

Not new to the HCI and multimedia literature is the attention to the relations between GISenabled computerized navigations and socio-political human practices. There has been also much discussion on the role that creative engagement and improvisation can play to break the rigidity of GIS-routing algorithms. Recently Daniela Rosner, whose work has been a source of our inspiration, has positioned maps as digital media to be altered and manipulated byhand to understand the subtle implications of algorithms as they move into social systems and daily life [50]. Only a few researchers, nonetheless, have tried to discuss on how many embodied social relations remain hidden under the surface of data generated by conventional mapping software. These social relations would be instead vital for all those people who live 
with activity limitations and participation restrictions. With Geo-Zombie we devise a new form of public participation in favor of people with disabilities, going beyond the traditional concept of engagement through entertainment. While laid-back fun "in fact" is often what people want in exchange for social commitment, we aim to transform the zombie apocalypse in an opportunity for people without disabilities to empathize with the condition of social exclusion suffered by disabled persons [29]. In doing so, we contribute with a study of digital participation through identification in a disadvantaged condition to a growing body of HCI and multimedia research on social inclusion where the idea is that even a selfish use of the technology, in order to achieve personal objectives or to personal entertainment, can be rerouted to let an altruistic purpose emerge (see e.g. [16,37,55]).

During field trials with fifty people (without disabilities) across Madeira (Portugal) and Cesena (Italy) Geo-Zombie encountered mixed reactions. On the one hand, some of the participants indicated they felt comfortable to extend their daily walking routines while providing a civic service, in some sense confirming, after Csikszentmihalyi, the vision according to which those activities are able to provoke a "mental state of operation in which a person performing an activity is fully immersed in a feeling of energized focus, full involvement, and enjoyment in the process of the activity" [8]. Others added that they had appreciated the nature of Geo-Zombie as resilient to a pure egoistic use. Finally, some other participants acknowledged that the activity of reporting on the presence of urban barriers and facilities had turned the everyday walking experience into an opportunity to augment their personal awareness of the surrounding environment. On the other hand, nonetheless, some people admitted to have experienced Geo-Zombie as deeply worrisome, due to the sense of impending disaster hanging over them on their usually relaxing walk. Many also indicated that Geo-Zombie in some sense prompted them to explore zones they did not want to travel, thus intensifying a sense of public obligation they refused. We used the contrasting feelings of the people we studied to understand potentials and limits of the forms of engagement GeoZombie makes possible in an effort to reroute personal resources toward an altruistic use. More generally all the discussion that follows in this paper documents an attempt to validate our conjecture around the shape that multimedia tools like Geo-Zombie can give to volunteering, civic services and social inclusion in the age of algorithms. Concept generation and discussion, focus group, experience prototyping, application design and implementation, and field trials (all discussed in the remainder of the paper) represent the different stages we passed to challenge our initial hypotheses and through which we would like to invite new reflections - and reactions in the HCI community. 
The work that is here presented significantly extends preliminary studies [43, 51] providing a detailed discussion on the design process and on the results achieved while investigating the concept of fighting exclusion through a multimedia application.

The reminder of this paper is organized as follows. Section 2 presents a background on: i) maps and algorithmic life, ii) urban data crowdsourcing, and iii) gamification as a strategy to motivate contributors. Section 3 provides details about the design and implementation processes we carried out, including: a) a concept generation phase where five mobile multimedia apps were proposed, b) a focus group that allowed us to select the two most suitable ones, and c) an experience prototyping phase where some users had experienced with the two selected multimedia mobile applications with the aim of refining them. In Section 4, field trials conducted with 50 users to contrast the two multimedia mobile apps mentioned before against a basic app, that requires users to collect data about the urban environment while providing no fun, are discussed, yielding both qualitative and quantitative results. Section 5 concludes the paper.

\section{Related work}

One of the most notable visions about maps posits them as apparently unbiased objects that instead represent a subjective point of view [27], and often also a mean for serving interests [27,62]. Maps can work "for me, for you and for us", becoming mechanisms for classifying purposes: "every map serves a purpose, every map advances an interest" [62]. For this reason, "all maps are selective", essentially amounting to "a purposeful selection from everything that is known", maintains Denis Wood, artist and cartographer [62]. In this perspective, it has been a long way before we have seen maps becoming an instrument for social inclusion. Since they work for a given purpose, only a targeted and privileged community usually benefits from them. Think, for example, of the use of maps to visualize crimes that deter people from visiting given neighborhoods [7], hence helping segregate specific parts of population, as pointed out in [50]. This utilitarian politics and practice of map-making has obviously resurfaced from traditional cartography into modern GIS-based multimedia tools, where only rarely the concept of social inclusivity represents an issue worthy of consideration. Nonetheless, in opposition to a plethora of conventional mapping software tools that build on Google Maps to provide various forms of fast, destinationoriented, pre-configured paths, recently some HCI authors have tried to rescue the emotional, cultural and symbolic values underlying digital cartography. Daniela Rosner and coauthors, 
for example, have shown that transforming hand-drawn digital sketches into walking paths, through the support of GIS routing algorithms, may open new possibilities for extending the work of mapping towards the notions of improvisation, creative engagement and autonomy [50]. Along a similar itinerary, Daniele Quercia and coworkers have integrated the crowdsourced dimension of beauty with a mobile mapping service that returns to customers routes that are not only fast and short, but also "emotionally pleasant" [44]. Our opinion is that, among others, one of the main merits of the approaches falling in this category is the fact that walkers become exposed to a sort of beneficial paradox: the longer they are engaged with experiences of emotional wandering using digital maps, the more they become surprisingly aware of the urban physical spaces surrounding them. A form of urban sensibleness comes with disorientation and surprise.

Less evocative in sense and imagination, more practical in use, several other multimedia software tools have been proposed in current years that rest on algorithms and GIS to compute routes that are tailored to specific personal characteristics and abilities. For those interested in speed, for instance, dozens of systems like WalkJogRun exist that offer personalized routes, facilitating training activities to frequent walkers and runners [57]. More notably, a civic sense of responsibility pervades those systems such as OpenTripPlanner [35] and Wheelmap [58]. The former allows to plan a multimodal trip (transit, bike and walking) in the Tampa area (Florida, US), also permitting to customers to search for wheelchairs accessible paths (if any). The latter is an online map that allows mobility-impaired users to search and find wheelchair accessible places throughout the world, as a result of the contributions that everyone can provide by marking (as either accessible or not) public places, like bars, restaurants, theaters and markets. These exemplar projects essentially follow on the heels of a wealth of digital approaches that try to solve community accessibility issues through crowdsourcing (see e.g. [33,36]). Interestingly, some of the more recent crowdsourcing initiatives in support of urban accessibility are backed by crowdworkers that contribute data in exchange of an economic compensation, typically using Amazon Mechanical Turk (see e.g. [23,24]). Unfortunately and paradoxically, although crowdsourcing is to be considered a powerful tool to fetch millions of data, it has been still difficult to reach a critical mass of data contributions of good quality in this specific field, as witnessed in many official reports [15].

A prominent role in this field is played by the concept of culture of participation. Culture of participation is typically intended as an inquiry to the participants to take more active roles within a given, already existing community. This can be done for either psychological well- 
being, or social integration or self-recognition or even a better credibility. Relevant are those recent studies maintaining that through this kind of culture "people have the opportunity to actively participate in personally meaningful problems in ways and at levels that they are motivated to do so" $[17,18]$.

Specifically, two different ingredients should be mixed together in the exact strength and dosage to establish a culture of participation: social creativity and richer ecologies of participation. With social creativity many authors intend that a wide range of participated contributions should be considered with the aim of solving a problem, that in nature comes from the interaction among people. Moreover, integral to this definition is the idea that hardware and software artifacts should be shared among those who contribute. The effect of all this is amplified if a richer ecology of participation is obtained through different levels of contributions that several persons can provide, each with its own role [4].

All this said, it is clear that a right mix of the concepts of social creativity and richer ecology of participation suggest a path at the end of which the goal of mapping the urban accessibility can be better achieved, through the activities, abilities and behaviors of different users, who have a different perspective while sharing a common purpose. People with disabilities and impairments, common walkers, municipal employees and others could hence provide a variety of accessibility data whose usefulness would be of great value if they all could appropriate such a culture.

Nonetheless, the issue of how to motivate those people remains partly unsolved. In fact, here we come with the key problem: Can the idea of linking access for disabled people with an explicit form of civic contribution actually work, if this contribution is to be provided (without any monetary reward) by the community of those who do not suffer any kind of impairment? What can digital tools do to facilitate free crowdsourcing of urban accessibility data, in the wider perspective of a culture of participation? What are the limits and potentials of civic recruitment through such modern technological developments as multimedia mobile apps and GIS-based technologies?

Along this line, the question of how to operationalize the use of interactive multimedia software tools in order to enlarge the community of those who can provide a contribution found an interesting answer in the practice of gamification. This concept has been widely investigated during the last decade (see e.g. [14,20,52]). It has been defined as exploiting "those features of an interactive system that aim to motivate and engage end-users through the use of game elements and mechanics" [52]. As such, gamification has been extensively exploited in a variety of different contexts, as it performs as either an intrinsic (i.e., altruistic) 
or an extrinsic mechanism that motivates people to do something. With extrinsic motivations, it is again intended that people that provide contributions are driven by an external separable outcome, ranging from an economic profit or an increase of reputation to effects of reciprocity or simply fun [25]. While gamification experts concur that both kinds of motivation are essential to gamification, it is desirable to rest upon intrinsic motivations whenever possible, because there are conditions under which if extrinsic motivations are added to an activity that already has an intrinsic motivation it can become demotivating. This is called "undermining effect" [11] and it is expected to happen, under some conditions, because rewards push people to engage in an activity for the sake of the reward rather than for the genuine enjoyment of the activity itself [59].

Indeed, our studies on gamification have revealed that one of the most tedious arguments in this field is that of intrinsic versus extrinsic motivations; rather it is more relevant to underline the fact that while some games exist that provide entertainment in exchange of human contribution, they all fall in the category of digital (i.e., Web) accessibility (see e.g. $[55,56])$. In sum, to the best of our knowledge, no gamification technique has been exploited to date in order to effectively crowdsource accessibility data for physical spaces.

Our Geo-Zombie project has therefore emerged from the need to examine how gamified multimedia GIS-based tools can be put to good use to improve data crowdsourcing for urban access. In doing so, we have built on the works about human engagement of Mihaly Csikszentmihalyi, Lucy Suchman, Susan Leigh Star and Bruno Latour. From the Csikszentmihalyi's vision of flow as an optimal state of intrinsic motivation for human commitment we have drawn the idea that "routine details can be transformed into personally meaningful games that provide optimal experiences" [9]. Hence our decision to motivate the practice of walking with game playing, as an act of personal cultural appropriation, also considering that "walking is the most trivial activity imaginable, yet it can be profoundly enjoyable if a person sets goals and takes control of the process" [9]. Inspired by the notion of invisible work described by both Suchman and Leigh Star, we have then tried to reframe the concept/metaphor at the basis of our game as an attempt to fight the stereotype according to which within a map drawn for some purpose "the interests involved in the work of mapmaking and map-using are effectively obscured" [53]. Instead, with Geo-Zombie any disinterested and altruistic activity of contribution clearly surfaces as the result of a process of personal identification with a disadvantaged situation from which to escape (that is the fear of zombies and the need to combat them). On this view, each map in Geo-Zombie becomes a reimagined place, both digital and material, where a singular phenomenon occurs. While the 
interests of the two communities of map-makers (normally abled people) and map-users (differently abled people) are reconciled, each single contributor has an opportunity to reflect on the ambivalent nature of her/his condition. In this sense, the value of a situated action taken in favor of the fragility of the humankind (fighting against zombies/signaling a urban barrier) takes the place of the well acknowledged hedonic valence of a horrific experience (see e.g. $[1,5]$ ). In sum, with the design of Geo-Zombie we have used all the concerns that derive from the Latour's intuition that certain values and political goals can be achieved through the construction and employment of given technologies to re-examine, and also to question, the mode through which gamified multimedia GIS technologies can shape the experience of contributing [30]. In the sections that follow all the phases of the development of Geo-Zombie are discussed while emphasizing the tensions, relevant to the HCI research, which underlie the different design decisions we made.

\section{Fighting exclusion: the design process}

Our design team sought to investigate this concept of fighting exclusion through a multimedia GIS-enabled social tool, engaging walkers in mapping architectural barriers. Our intent was to understand if our intuition to exploit zombies genre as an apotropaic medium in improving civic engagement can emerge from different multimedia mobile apps ideations. We wanted to test if our conceptualization of fighting exclusion by mean of a pervasive use of zombies and maps could become a concrete step towards social inclusion. Students, researchers and professors took an active role in the design process as explained in the following.

\subsection{Concept generation}

We started the design process with a concept generation session recruiting 13 international students from the Master in Human-Computer Interaction (8) and the Master of Entertainment Technology (5) within the Carnegie Mellon - Madeira University International Program. This was motivated by the fact that the typical group of users targeted for the applications of interest is intended to be that of young adults. In fact, our idea is that it is more likely that youngsters wander about the city and discover new paths with their barriers/facilities.

We began by illustrating our goal of mapping urban accessibility via crowdsourcing and crowdsensing with the aim of providing multimedia inclusive paths and services. Secondly, 
we shared our concern about the effectiveness of the system due to the dimension and the characteristics of the community of users. The use of entertainment elements appeared as the obvious solution to enlarge the community interested in collecting data about urban accessibility. Based on insights we gathered from this design session, we developed the following three design concepts related to motivation, movement and community.

- Motivation through Aesthetics: from discovery to fear. The discussion highlighted the need to explore aesthetics in game as an engagement tool [26]. In fact, aesthetic components are what make the experience pleasurable: the power of these components is in the intrinsic motivation that the experience recalls in the player. They were: discovery (game as uncharted "territory"); pastime vs challenges, and feelings (including fair). Mark argued that "civic engagement can be a strong intrinsic motivation but it needs to be reinforced using entertainment and fun" in order to keep motivation alive. Very unexpected for us was the feeling reported by Anita. Thinking to a blind person, she said: "it must be scary walking in an unfamiliar territory with the continuous feeling of imminent danger but normal people cannot face that kind of fear... they, we, just unconsciously ignore it". This looked very close to our vibes to use zombies and the horror paradox [1] to inspire our mobile app.

- Movement: physical and civic. The role of movement emerged from the discussion. In particular, Nikolas emphasized: "We need to move! We cannot stand still and wait! Step by step we can make a difference". Movement brings in mind two different meanings: movement as the consequence of body moving and movement as heterogeneous group of people tending toward or favoring a common goal. In the first sense, movement can result in a healthy activity or accompanied with a particular task, such as getting to work or running errands. In the second sense, movement is a form of civic engagement.

- Breaking boundaries in community. The students we interviewed described several possible targets, obviously including people with disability, but going beyond considering other groups of people. Mary, a young mom, shared with us: "playing around the neighborhood could be a good alternative to watching TV', suggesting kids as a possible target community. The most popular idea was to focus on young adults and teenagers, people that usually walk, wander, explore and like playing games. 
As a result of the definition of these three design concepts, many multimedia mobile apps proposals came into mind of the students involved in the concept generation. After a discussion, we sketched the five multimedia location-based applications more suitable for our goal. Each application embodies the three design concepts, focusing on different aspects.

1. HINT! (Discover your Hidden INTerest!). HINT! was conceived addressing several of the concepts emerging during the discussion documented before in particular: body movement (players are assumed to walk), pastime vs challenges (completing a puzzle), breaking the community boundaries (not only people with disabilities are target for using HINT!). Specifically, HINT! works as follows: there is a hidden image under the area where someone is walking through. The more a player walks and collects data, the more $\mathrm{s} /$ he obtain pieces of the puzzle in order to understand the subject of the picture. The idea is to have different sets of pictures related to a specific topic (e.g. nature, animals, movies, anime, culture, and so on) to enlarge the set of interested people. As soon as players guess the content of a picture, they earn points.

2. Geo-Zombie. Geo-Zombie was designed basically based on the two following concepts: a) arousing a feeling of fear with the aim of implementing the horror paradox discussed in Section 2, b) encouraging physical movement under the pressure of escaping from zombies, and c) resorting to an entertainment application to enlarge the base of those who will contributed. Geo-Zombie is about staying alive and avoiding being eaten by zombies. One is exploring the surrounding with the aim of acquiring ammunitions to shoot the zombies. Meanwhile, players are requested to crowdsource accessibility data in change of weapons and ammunitions. Hence, walkers are pushed by zombies to wander, distracting from their planned, destination-oriented daily routes. Zombies are generated randomly and situated around the walker's GPS location with a variable distance. Zombies walk the most efficient path (provided by GIS) to catch the player. Movement is not only physical: walkers that kill zombies are deeply connect with the concept of imminent danger, which can be fought resorting to appropriate civic actions.

3. Geo-Minesweeper. This application was suggested as it relies on the following concepts: a) provoking the feelings of challenge, needed for discovery and fear (while walkers demine the areas all around them), and $b$ ) enjoying the fun of avoiding a virtual mine explosion. A player has to complete a path from the starting point to the final destination while demining when necessary to have a free path. This application extends the tradition minesweeper game in the sense that at each urban barrier corresponds a mine. The virtual mine is hence a real urban barrier: if it is not documented, it blocks walkers to reach their 
destination. This wants to recall to the user the difficulties experienced by people with disabilities facing unknown barriers. Every time a walker passes near a barrier is requested to document it. The repetition of this action has the important side effect of improving the quality of data through the validation of the same urban element by different users.

4. Geo-TreasureHunt. Geo-TreasureHunt was conceived considering: a) kids as interesting factors to enlarge the community of contributors, and b) kids' physical movement as a beneficial side-effect for wellbeing. This application implements a treasure hunt competition among children. The subject of each question of the treasure hunt is concerned with the idea of making kids moving to a location where a physical barrier is placed, with the intent to signal it. Obviously, kids are supposed to be accompanied and supported by an adult.

5. Basic app. The decision of adopting a basic application consisting in a simple tool to collect data about urban accessibility, while walking in the city, was taken for comparison against the other more sophisticated ones. This application leverages on a really genuine intrinsic motivation to contribute since no other factors are exploited here to convince people to participate.

\subsection{Focus group}

At this stage, we organized a focus group with experts in order (i) to assess the pros and cons of each game strategy in relation to our goals and (ii) to narrow the selection of the best suitable multimedia mobile apps, to bring forward to the deployment stage.

The focus group was organized at the Madeira Interactive Technologies Institute (M-ITI), it lasted around two hours and involved ten participants including $\mathrm{PhD}$ students, researchers and professors at the institute with familiarity with gameplay, game design and interactive technologies. The proposing researcher led the discussion through a presentation of the five multimedia mobile apps ideation using a series of slides.

During the focus group the Geo-Zombie and HINT! multimedia mobile apps emerged as the most attractive yet engaging ones, out of the five multimedia mobile apps presented. Both Geo-Minesweeper and Geo-TreasureHunt got some negative reactions. In particular, GeoMinesweeper was considered as an aggressive game by some components of the focus group. Anirudh observed: "Mapping a barrier is like mining the city and impairing other walkers". Regarding Geo-TreasureHunt, the need to be organized by teachers and/or parents makes the 
game not suitable for daily playing, and this is a strong limit in obtaining an effective enlargement of the mapping community. John, a researcher, said "this game requires a lot of work: it would be interested for kids but I doubt that it can be played more than once".

The Geo-Zombie was perceived positively, although the zombies are very popular in many games. We got some enthusiastic comments from younger participants, who maintained "It can work!".

HINT! was appreciated but some participants proposed to implement the hidden puzzled image as a gift voucher that is a coupon which is assigned to players as soon as they signal barriers/facilities and complete the puzzle. In other words, as soon as a player provides a report, s/he gets a piece (fragment) of the puzzle (which is indeed the image of the voucher). At the end of the game that voucher could be spent in shops located in the game area.

We decided then to proceed to experience prototyping Geo-Zombie and HINT!, in order to assess if transferring the concept to a physical experience prototype could reveal some unexpected findings.

\subsection{Experience prototyping}

The experience prototyping is a technique borrowed from experience design and service design disciplines in order to test the flow of an experience or service in physical space [3, 28]. Such prototyping technique helps to refine the concept and the overall design of the experience before any investment is made in detailed coding and hardware prototyping.

In order to carry out an experience prototype of both Geo-Zombie and HINT! multimedia mobile apps we engaged four users who in turns tried both apps. For Geo-Zombie, we prepared paper wireframes of the phone interface (see Fig. 1.a) and physical zombies paper puppets to chase the users; to shoot the zombies we used a plastic gun recharge with water (munitions), as shown in Fig. 1.b. For HINT! we prepared the paper wireframes of the screens and a final puzzled voucher for the players (see Fig. 1.c).

Subsequently we defined a task to execute, and shadow the users and took notes while they were playing with the multimedia mobile app. At the end of the experience, a short interview was conducted with each participant. Our participants were all employees (faculty and staff) of the research center of Madeira Interactive Technologies Institute, two female and two male, ranging from 25 to 45 years old, with some experience in technology and gameplay. In particular, Miguel is a 45 years old Professor, Ana is a 25 year old Master 
student, José is a 30 year old $\mathrm{PhD}$ student and Teresa is a 33 year old accounting clerk and administrative manager.

The task of the users was an ordinary one, something they could be done in their daily lives, considering the gameplay as an extra entertainment. For the purpose of our games, we asked the users to compare the price of two products in the university canteen with the closest bar near the university. As suggested during the focus group, we asked users to map only one type of barriers/facilities in each experience prototyping round, preventing users from being confused from paying attention to many different types of urban elements. In particular, during the HINT! experience prototyping, we asked users to map steps and stairs, while during the Geo-Zombie experience prototyping, we asked for zebra crossings.

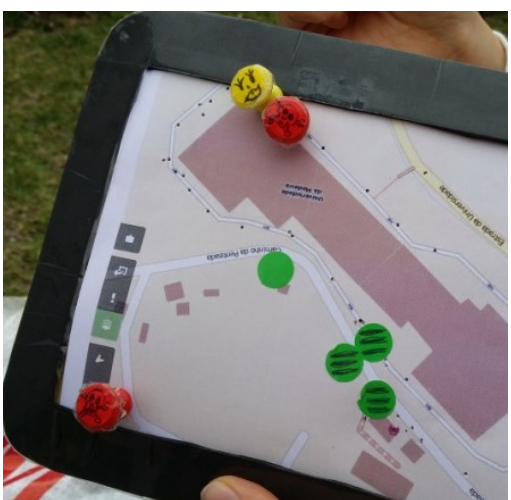

(a)

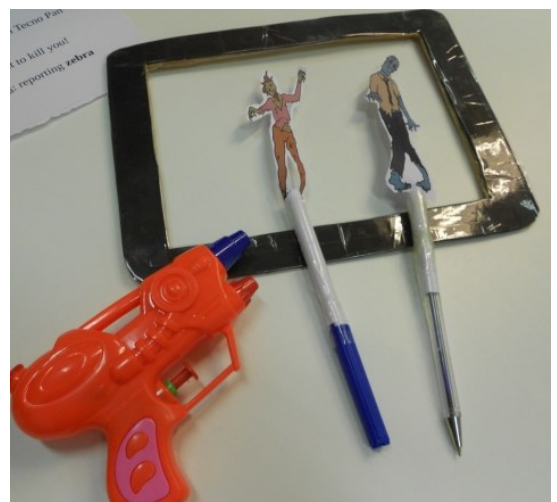

(b)

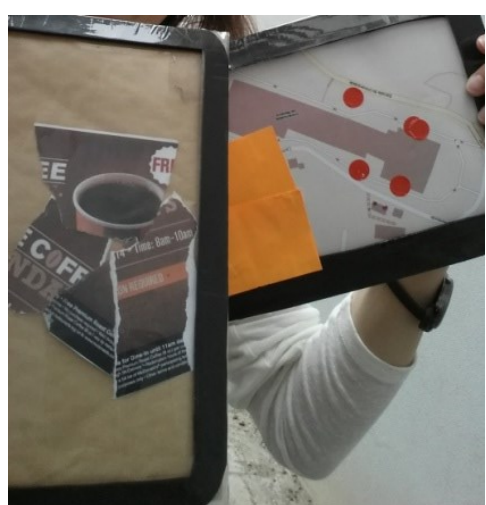

(c)

Fig. 1 The experience prototyping equipment

\subsubsection{HINT! experience}

The HINT! experience prototyping involved four walkers playing the game while executing a simple task, who had to check the price of a specific ice cream at the university canteen and at a bar nearby. Overall, the players seemed less excited to play HINT! than playing the Geo-Zombie multimedia mobile app. In fact, they did not run or increase their walking speed. This was all somehow expected, since the HINT! multimedia mobile app was designed to appeal to an audience interested only in the reward. While mapping barriers, the user can see and retrieve vouchers fragments, which are provided in pieces, making each single piece available to users for each barrier/facility mapped. Fig. 2 shows two pictures of two users busy in collecting voucher fragments during the round. Getting pieces of the voucher seemed to be a strong motivator for three players out of four. Miguel, was not interested in the voucher. Furthermore, he was also disappointed by the type of voucher gift 
that he received at the end. He clarified in the interview that it is important for the voucher to be personalized and connected with the user interests. On the other hand, two other players were excited to get some free voucher of any sort. One of them confirmed: "I prefer playing HINT!. It was kind of easy to do, without someone that was trying to catch me... and I like the voucher thing. Geo-Zombie was too much of a game to have in my daily routine". Teresa also found the voucher motivating the gameplay "The voucher is a big motivation... maybe it can be used also in the zombie game...".

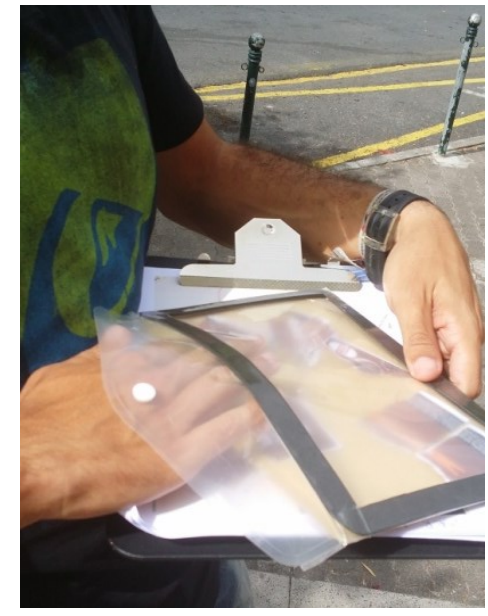

(a) collecting fragments

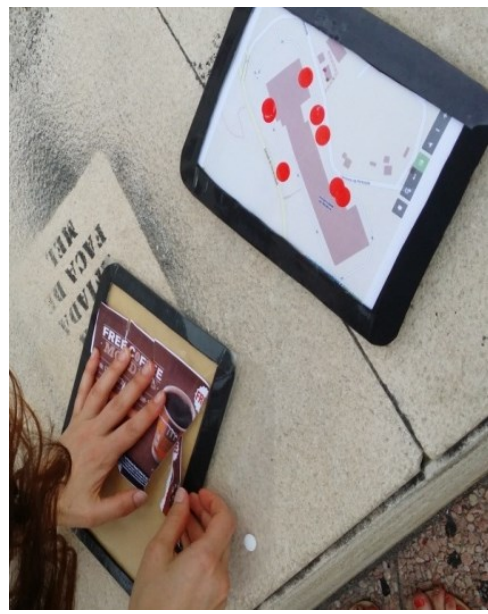

(b) compliting the voucher

Fig. 2 Two pictures taken during the HINT! experience prototyping.

A note to mention was the Miguel's words: "I feel very motivated about mapping urban elements just for my interest in exploring the area and discovering them". He also added that the idea of contributing to the bigger good can function as a rewards on its own (intrinsic motivation) besides getting the voucher (extrinsic motivation). He would do it even without a voucher return because of his own pleasure in mapping the territory and helping people with disabilities in avoiding barriers. In particular, he claimed: "I think the important thing is to become aware with the space around me because this is the space that I had around for ten years and I was surprised about how many zebra crossings there are and zebra crossings are so big so I cannot imagine to look for another things, like a step or an obstacle in the sidewalk".

Another player, José, had a similar opinion about the importance of mapping urban accessibility: "Mapping in itself is a strong motivation, more than the gameplay, because people become more aware about the space they are surrounded by and it is very important". 


\subsubsection{Geo-Zombie experience}

The second experience prototyping involved the same four players engaged in the HINT! round. In this particular case, comparing prices of coffee from two bars across the campus. Fig. 3 shows two pictured taken during the round. From our observations and interviews we can confidently affirm that during the experience prototyping of Geo-Zombie all our four users had a lot of fun.

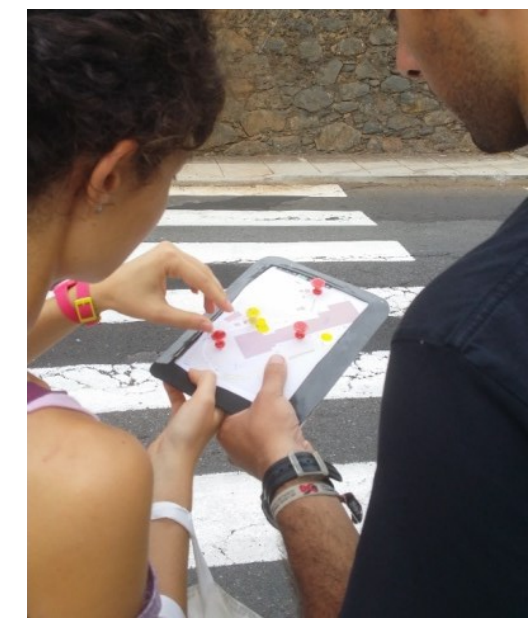

(a) Mapping activity

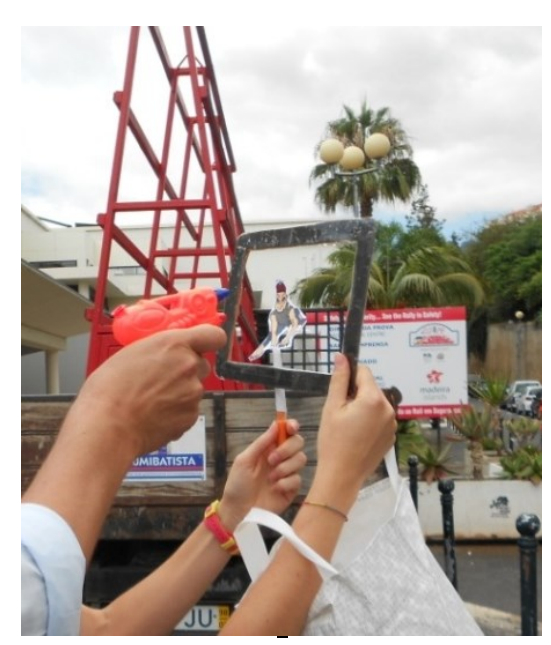

(b) Shooting the zombie

Fig. 3 Two pictures taken during the Geo-Zombie experience prototyping.

They enjoyed the multimedia mobile app and two of them went on playing, after their task was complete to map more urban elements in order to acquire more points and ammunitions. Two of our users surprised us by entering a building as an escape technique to hide from the zombie. This technique was successful for survival (the zombie follows the user's GPS coordinates which cannot be traced indoors), and allowed them to reach faster barriers/facilities close to the exit in the opposite site of the building. Another unexpected behavior of one user was to switch strategy after some gameplay: instead of running away from the zombies, he preferred to chase them. This strategy brought about some implementation issues. First, Miguel said: "I found the game become too easy, it needs more zombies". Such comment made us focus our attention on the number of zombies, hence on the level of difficulties that different players would enjoy. The same user also reported: "When I had the gun then I changed the strategy so I really want to kill the zombie but I was 
still interested in finding zebra's crossings because I was not aware that there are so many of them around this place".

Such comment made us realize that being engrossed in the game, may also distract players from mapping barriers/facilities.

Other considerations of the same players regarded the travelling speed of the zombies. "Maybe zombies with different speed would make the game more exciting", a user suggested. All of the Geo-Zombie players were so immerse in the game to increment their walking speed to escape from zombies. In some cases, they even started to run. A player explained: "There were too much zombies around me, I felt trapped and I started walking faster without realize that!".

\subsection{Implementation}

We implemented HINT!, Geo-Zombie and the basic app as multimedia hybrid apps that can be built ad run for Android, iPhone, Windows Phone and other platforms [38]. We tested the three multimedia apps on different smart devices running Android 4 or higher and iOS 7 and higher. Those smart devices feature a touch screen, a build-in GPS receiver, a camera, an accelerometer, a gyroscopic, and a Wi-Fi or cellular data connection capabilities. We wrote the software using web technologies (HTML, CSS and JavaScript) and the Cordova plugin APIs [6]. We used Google Maps JavaScript API v3 [19] to manage the position of the user (and zombies). The back-end part has been implemented as a RESTFul Web Services using Java 7 and the Spring framework. Data are stored in a PostgreSQL DB [41], integrated with PostGIS [40] to enable support for geographic objects.

In particular, HINT! and Geo-Zombie include three functions: (1) Walk, (2) Report, and (3) Play, while, the basic app only: (1) Walk and (2) Report.

Specifically, The Walk function allows people to start the game round, taking the GPS location and visualizing the current position in the map (see Fig. 4). It is in this moment that the system starts to track the user's trace.

Using the Report function instead, people can provide urban accessibility data, trust gaining game points. To report an accessibility element, walkers need to choose the right category from a defined list and send the server this information together with their GPS position (see Fig. 5). They can also supply additional information (like a brief description, notes and photos) so as to obtain even more points. With the aim of validating collected data, all the apps also provide a notification to players as soon as they walk in an area where 
barriers/facilities were already documented by others, to confirm their presence. In case the report a new walkers provides complies with the majority of the already received reports, the user earns points.

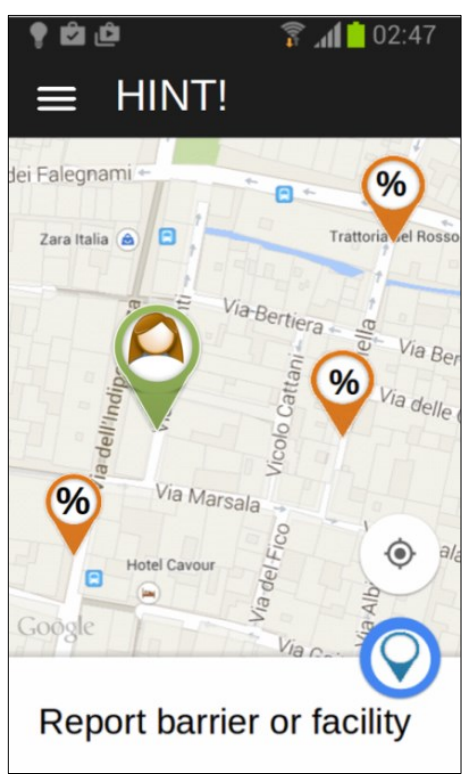

(a) HINT!

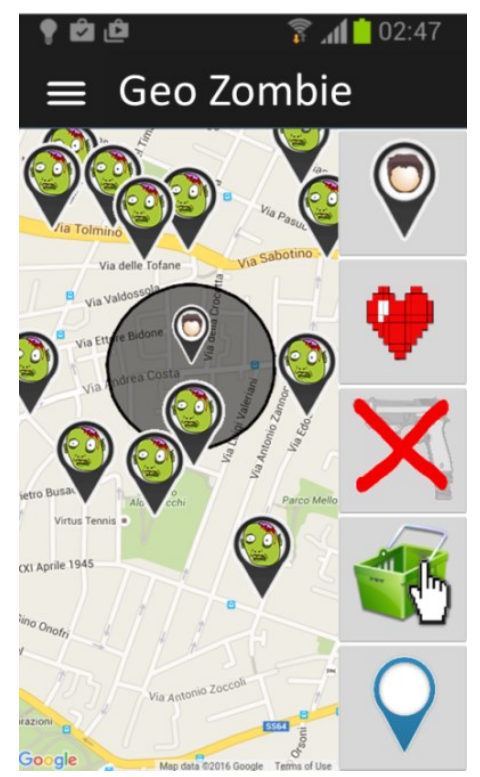

(b) Geo-Zombie

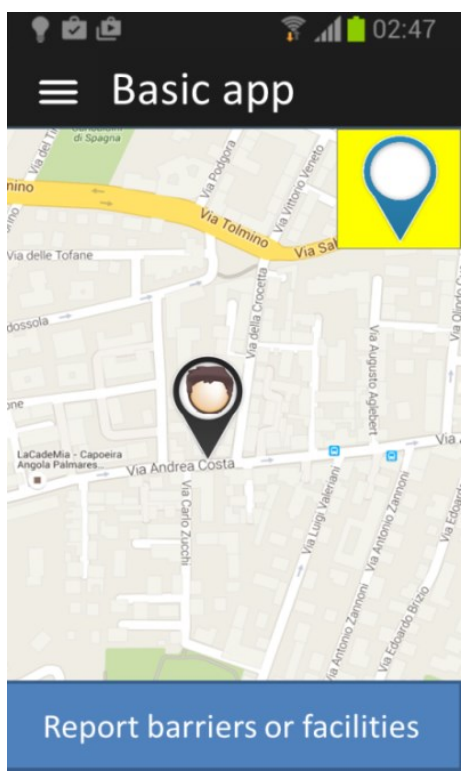

(c) Basic app

Fig. 4 Screenshots of the Walk function

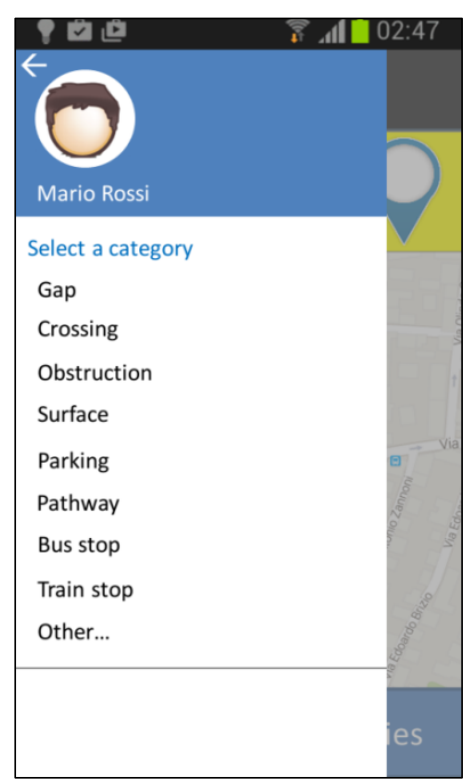

(a) Choosing a category

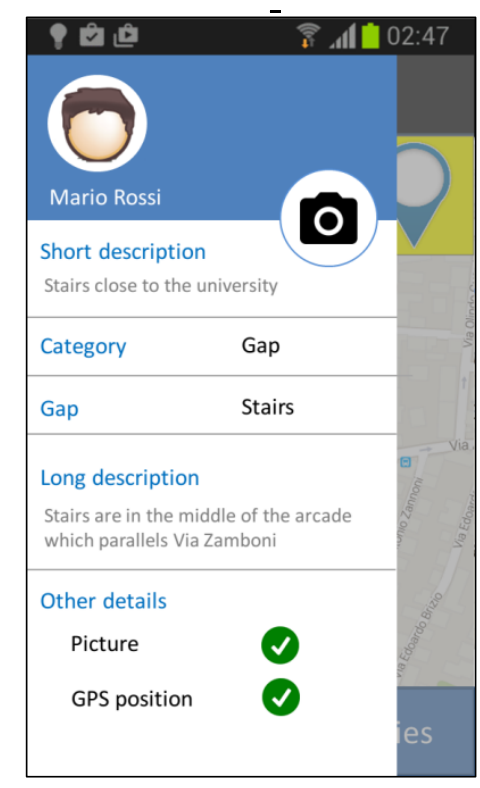

(b) Reporting a barrier

Fig. 5 Screenshots of the Report function 
As to Play function, it is implemented in different way depending on the chosen app (either HINT! and Geo-Zombie).

We already mentioned that HINT! is based on a reward (the voucher), hence, the Play function allows one to collect pieces of the voucher/puzzle and then reorder them, to complete the puzzle (see Fig. 6). New shops (and so vouchers) become available based on the paths a walker follows. Using the Report activity people can document barriers/facilities and obtain points to spend.

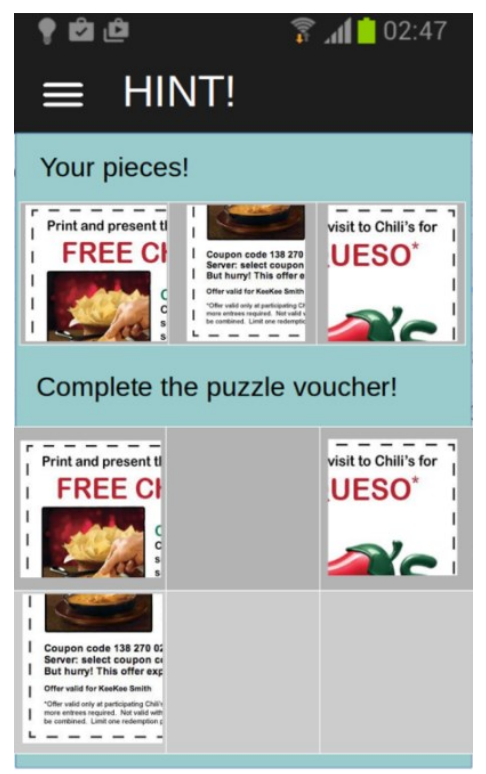

Fig. 6 A screenshot of the Play function (HINT!)

The Play function comes after Walk and Report. In particular, the system has to follows two steps: (1) gets the GPS position of the player; (2) randomly distributes zombies over the game area. Google Maps based routing algorithms are used to let the zombies approach to the user, through the shorter path.

With the Report function, barriers/facilities are mapped and points are gained to be spent for ammunitions, weapons, and energy. To map a barrier/facility, the player just needs to push the button with the light blue marker (Fig. 7) and use the Report function.

Thanks to the Walk function, people can follow their current position in the map while avoiding zombies. Zombies walk at different random speeds with the aim of catching the player, following the shortest path. When a zombie is close to a user (approximately ten meters), the visualization mode within the app switches from maps to reality (camera view in Fig. 7) suggesting the player to shot the zombie before its attack. From a technological 
viewpoint, it is worth mentioning that we have drawn zombies within an augmented reality setting using Wikitude [60].

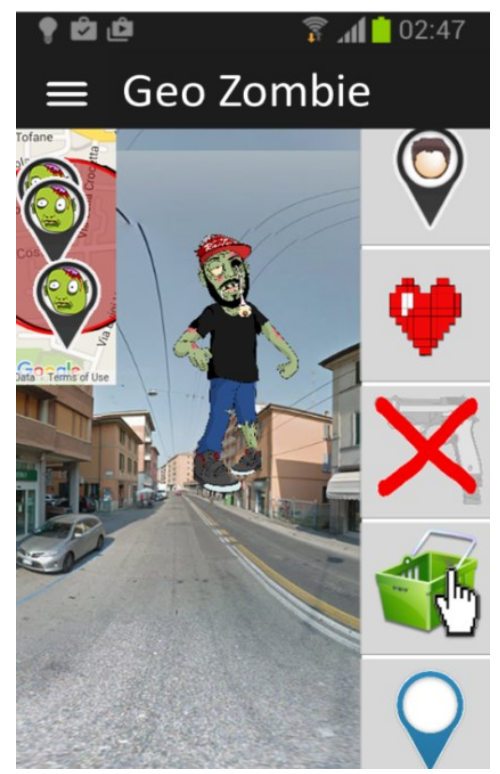

(a) Zombie approaching

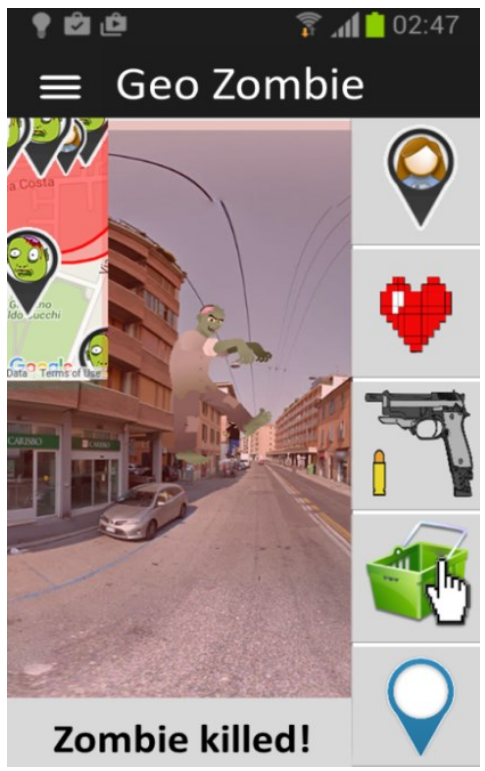

(b) Shooting at a zombie

Fig. 7 Two screenshots of the Play function (Geo-Zombie)

\section{Fighting exclusion: in practice}

The following Section is split over two parts as suggested in [61]. In Subsection 4.1 the context of the experiment (also termed planning) is described, while Subsection 4.2 provides both quantitative and qualitative results.

\subsection{Planning}

After developing Geo-Zombie, HINT! and the basic app, we decided to recruit students in field trials who denote a target of avid players and walkers. For this reason, we ask to a class of fifty students (39 males and 11 females), enrolled on the Multimedia System Technologies course of the master degree in Computer Science and Engineering (University of Bologna, Italy), to experience the three multimedia mobile apps. The students attend courses in Cesena, but they live in different cities, including Bologna, Cesena, Faenza, Rimini, and Ravenna. These cities have different densities and layouts, relevant factors in examining the variety of city urban environments. Students were free to decide if participate or not to one or more test fields. In fact, during the course, we asked the students to complete at least 6 class assignments on the 12 presented (as part of the final evaluation). We made the apps available 
to the students of the course and we monitored their use by logging data in a PostgreSQL DB [41].

We studied approximately fifty students' use of HINT!, Geo-Zombie and the basic app over three weeks (a week per app). The first week they could mapping barriers using the basic app, the very simple multimedia app created to just collect data about facilities/barriers in the urban environment. Using such an app, the user could easily map urban elements, sending information about the GPS location and the typology. To obtain the class assignment done, students just needed to download the multimedia app and map at least an urban element. The second week, they would be able to obtain a voucher with HINT!, that is, a valid class assignment, mapping at least 5 barriers/facilities. The last week they managed to fight with zombies using Geo-Zombie. As with the basic app, to obtain the valid class assignment, we asked for at least one urban element mapping.

After each field trial week, we involved students in filling a survey related to the trial app. The survey was composed of 16 items: 5 items related to the goals of the system, 10 items connected to the specific tried app, and one open question for comments and hints. We use the Likert scale [2] approach in ranking the firsts 15 questions based on a 5 values symmetric agree-disagree scale, in order of strength. In particular, the adopted scale was as follows: strongly agree, agree, neutral, disagree, strongly disagree. Moreover, we gave participants the possibility to provide personal comments. Obtained data was analyzed from both (i) a quantitative viewpoint and from (ii) a qualitative perspective, obtaining interesting results.

\subsection{Results}

In the following Subsections, we provide, respectively: (4.2.1) a numerical analysis of the effects provided by the gamification concept (included a repeated measures ANOVA test), (4.2.2) a time and space dynamics analysis of our data and, finally, (4.2.3) a qualitative analysis.

\subsubsection{Quantitative results}

On a quantitative perspective, a first element of interest is the fact that almost all the students of the class accepted our proposal and took the decision to participate $(95 \%$, approximately). While, the number of participants was reduced with the HINT! (47 students), a so large amount of students accepting to try to use both the basic app (48 students) and Geo-Zombie (48 students) version of our mobile application was not expected. Nonetheless, 
not surprising was the fact that the number of barriers/facilities documented using the basic app (95) resulted much lower than the amount of those reported through the use of either the HINT! (311) or Geo-Zombie (286) apps. Indeed, this result can be easily explained with the consideration that both HINT! and Geo-Zombie apps supply concrete motivations to users to continue to play, while no external motivations exist that can encourage people towards a long term use of the basic app. All these numerical results are summarized in Table 1, along with the fact that the number of reports obtained with HINT! is comparable with those obtained with Geo-Zombie (311 vs. 286).

Table 1 Numerical comparative results

\begin{tabular}{|l|l|l|l|}
\hline \multicolumn{1}{|c|}{ Descriptive statistics } & \multicolumn{1}{c|}{ HINT! } & \multicolumn{1}{c|}{ Geo-Zombie } & \multicolumn{1}{c|}{ Basic app } \\
\hline N. of users & 47 & 48 & 48 \\
\hline N. of reports & 311 & 286 & 95 \\
\hline Arithmetic mean & 6.60 & 5.95 & 1.97 \\
\hline Median & 5 & 3 & 1.5 \\
\hline Geometrical mean & 5.57 & 3.19 & 1.67 \\
\hline Standard deviation & 3.56 & 8.60 & 1.28 \\
\hline Variance & 12.67 & 73.96 & 1.63 \\
\hline Range & 6 & 35 & 18 \\
\hline
\end{tabular}

An additional interesting information portrayed in Table 1 is that almost all those users who played with HINT! made approximately the same number of reports (6 on average), while those who enjoyed Geo-Zombie provided mixed results. Precisely, analyzing the frequency, 25 of them contributed with from 1 to 5 reports, while the others 23 contributed with more than 5 reports. These results are confirmed by: i) the high value of the standard deviation from the average number of reports per user measured for those who used GeoZombie app, and ii) the low value of the corresponding metrics for HINT!. This underlines a substantial difference between exploiting either fun/entertainment or a more concrete reward to motivate people to crowdsource accessibility data.

In fact, while with HINT!, almost the totality of engaged students was driven to reach the threshold of 5 reports to have the assignment passed, with Geo-Zombie only those students intensely participated who felt emotively engaged by the multimedia mobile Zombie experience. Based on this preliminary result, one could guess that the amount of civic 
contributions that can be collected by offering a concrete reward can be similarly obtained with a consistently lower amount of participants (approximately a half, in our case), provided that an emotionally captivating gamification strategy is adopted. As a final consideration on Table 1, it is worth noting that median, geometrical mean, variance, and range values confirm that both HINT! and Geo-Zombie have aroused interest (and so more reports) than the basic app did.

Up to this point, we have provided a wealth of descriptive statistics that highlight the supremacy of the gamified variants of our application. Nonetheless, a more detailed and statistically well-grounded evaluation is needed to compare the three different approaches. In particular, we are interested in understanding whether the larger quantity of reports produced by players who used such gamified variants of our application either is a result of the gamification effect or is simply accidental. Specifically, we carried out a repeated measures ANOVA test to confirm that the probability that the given players produce accidentally a higher number of reports, with the gamified variants of our application, is lower than a given threshold value: $p \_$value $<0.05$. Hence, the null hypothesis $\left(\mathrm{H}_{0}\right)$ and the alternative hypothesis $\left(\mathrm{H}_{1}\right)$ follow.

$\mathrm{H}_{0}$ : There is no difference in using either a gamified or a non gamified version of our application, in terms of produced reports per user.

$\mathrm{H}_{1}$ : There is a difference in using either a gamified or a non gamified version of our application, in terms of produced reports per user.

As already said, based on the consideration that our data satisfy all the assumptions on which a repeated measures ANOVA test is based (included the distribution of the dependent variable in the three related groups as approximately normal), we developed such a test that supplied the following result:

$$
\text { p_value }=0.04\left(F \_ \text {statistic }(2,94)=3.32\right) \text {. }
$$

Based on this result, the null hypothesis can be rejected and we have a further quantitative confirmation that introducing gamification has a significant (and not accidental) effect in the number of the reports provided by contributors. 


\subsubsection{Time and space dynamics}

To continue this discussion under a different perspective, Fig. 8 shows the time dynamics through which the reports were obtained. Interesting here is only the fact that with both HINT! and Geo-Zombies a great part of the reports were collected within three/four days. This is easily explained based on the consideration that after a few days of urban exploration, almost all the barriers/facilities, at a walking distance from the Computer Science Department, can be easily reached by quiet walkers.

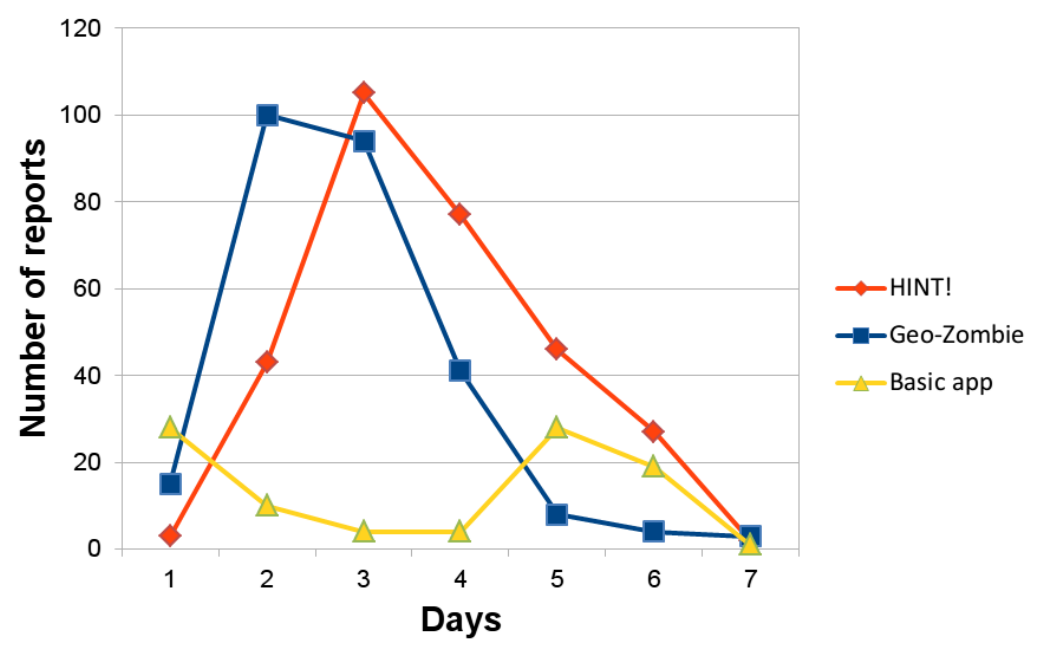

Fig. 8 Number of report: evolution over time

Final and important considerations on a quantitative side can be derived also from an analysis of Fig. 9. Each of these figures shows the geographical distribution of the barriers/facilities, present in one of the areas where our experiments took place, reported as either signaled (green squares) or not signaled (red dots) with respectively the Basic, HINT! and Geo-Zombie mobile app. To begin, we can observe the quite expected result that a lot of urban barriers/facilities remain undocumented with the basic app (Fig. 9.c).

Even more interesting is the result that the number of barriers/facilities detected with Geo-Zombie outperforms the amount of those intercepted using HINT! (Fig. 9.a and 9.b). This fact has a very highlighting explanation that is as follows. If one considers the specific geographical area shown in our figures, s/he can discover that the Department of Computer Science is located in the top left sector of the map (purple marker in the figures), while both the bus and the railway stations are placed in the bottom right section of the map (light blue marker in the figures). 


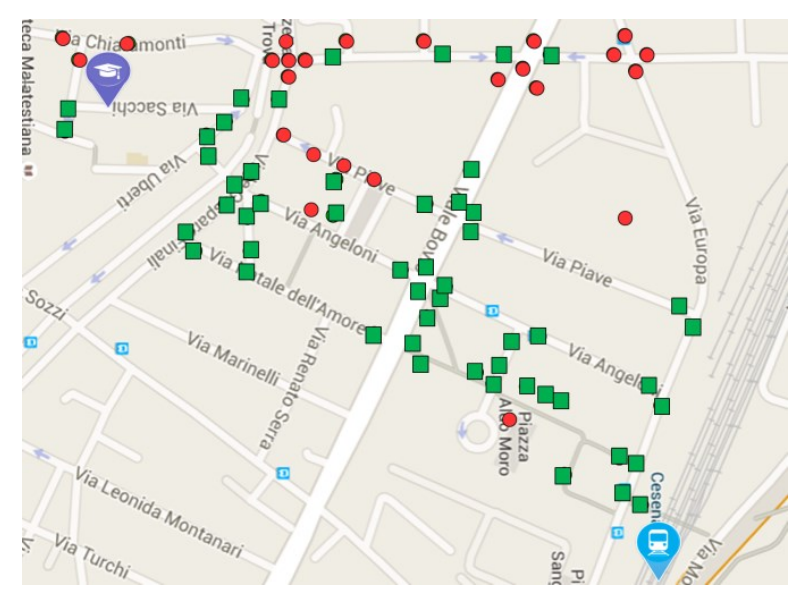

(a) HINT!

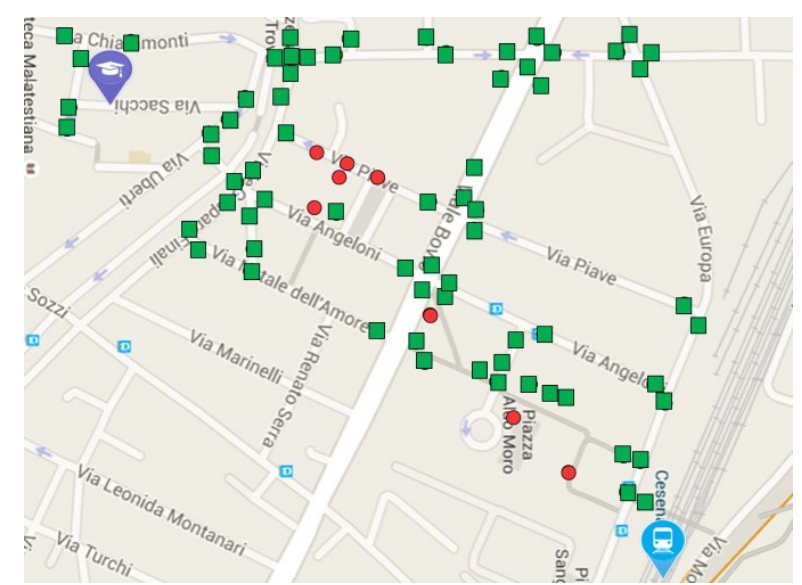

(b) Geo-Zombie

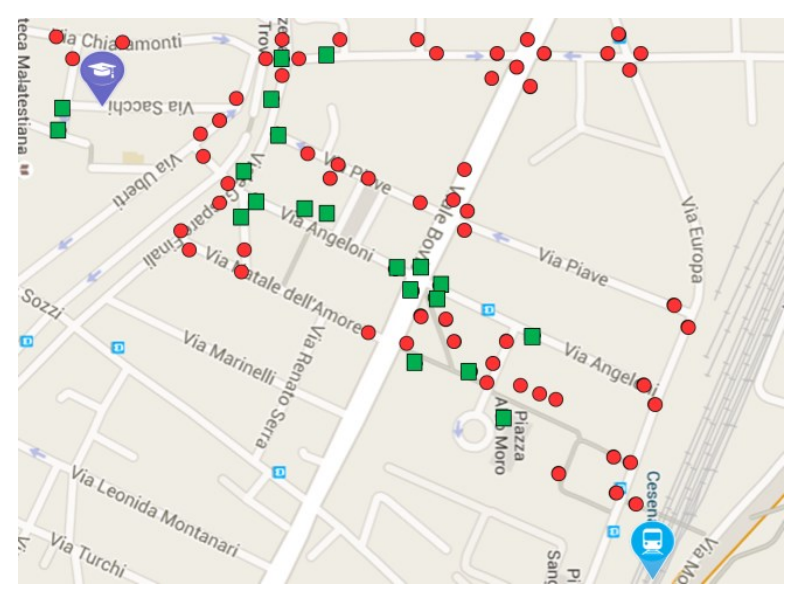

(c) Basic app

Fig. 9 Existing barriers/facilities mapped (green squares) and not mapped (red circles) by the students

What happened with HINT! was that our students have privileged walking along their usual route from the stations to the Department (and back). This route amounts essentially to the line that transverses Fig. 9.a from its bottom right section to the top left one. With Geo-Zombie, instead, users favored a form of non-destination-oriented walking over efficient, familiar routes (see Fig. 9.b). This is witnessed by the path connecting the top left sector of the map with the top right one, whose barriers/facilities were signaled only by those walkers who used Geo-Zombie (Fig. 9.b).

\subsubsection{Qualitative results}

We asked all the 48 participants to fill a survey and we have conducted some few short interviews in order to understand from a qualitative view point their feeling about the use of 
the three apps. Some interesting - and sometimes contrasting - notion emerged related to different investigated design concepts.

Not surprisingly, the first conception emerged from the interviews was the strong relation about the enjoinment of the multimedia app and the engagement in the mapping activity. Fig. 10 shows the students' answers to the item "The mapping activity was boring", for each trial apps. As expected, comparing the results obtained from the three apps, we can reveal that almost all the interviewees found the basic app too boring to be used frequently despite its social value, while different motivations were provided in support of the use of either HINT! or Geo-Zombies. Interesting results are related to HINT! if integrated with the quantitative data. The app is considered "neutral" (neither boring nor funny) by 20 students (42\%), but this feeling didn't compromise their engagement in reaching voluntarily the voucher (a valid class assignment). This suggests that the most of the students were engaged by the curiosity in discovering the voucher. For example, Matteo affirmed:

"I found HINT! more engaging that the basic one, it wasn't funny, but I wanted the reward so I continued to report elements until I have achieved it!"

Analyzing the data in Fig. 10, it is possible to notice that 30 students (64\%) found Geo-Zombie not boring (answering "disagree" or "strongly disagree"). This result probably is related to the enjoyable sensation of positive fear perceived by students. In fact, Marco said:

"Playing with Geo-Zombie was really exciting! The idea of so many zombies ready to catch me was scary but at the same time, challenges! I liked that feeling!"

According to this concept of positive fair, Lucia maintained:

"There was immediately a zombie there, and I had to kill him or I would die, the game made me feel differently... excited"

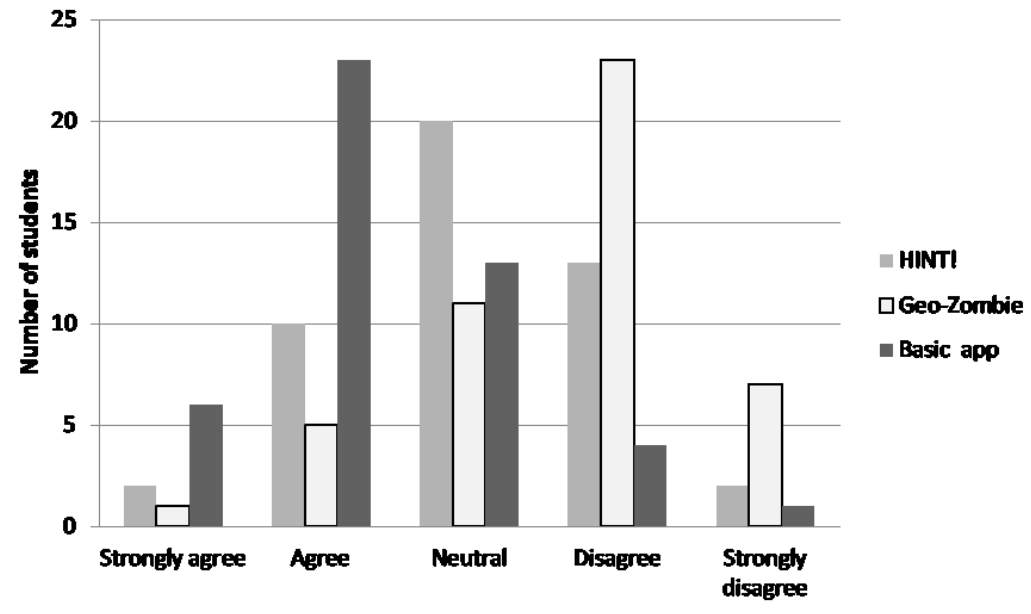

Fig. 10 Answers to the survey item "The app was boring" 
Data in Fig. 11 show another interesting result strictly related with the concept of movement (physical and civic). In particular, with the basic app, students were not interested in changing their routine and their daily paths, and consequently, in expanding their mapping activity. Different results were obtained with HINT! and with Geo-Zombie. The use of gamification strategies seems to motivate participants in enjoying detours from their normal daily routines. In particular, it is very impressive that, by using Geo-Zombie, more than $50 \%$ of the students ( 25 of 48 - 15 "agree" and 10 "strongly agree") explored new paths, broking their routine. This is probably a consequence of the game flow [54] that allows players to forget about their daily habits in order to enjoy the game. Pietro claimed:

"For the first time in three years I took a different route to reach the railway station as I had Zombies behind me, and I discovered a very interesting route, full of small food shops!!”

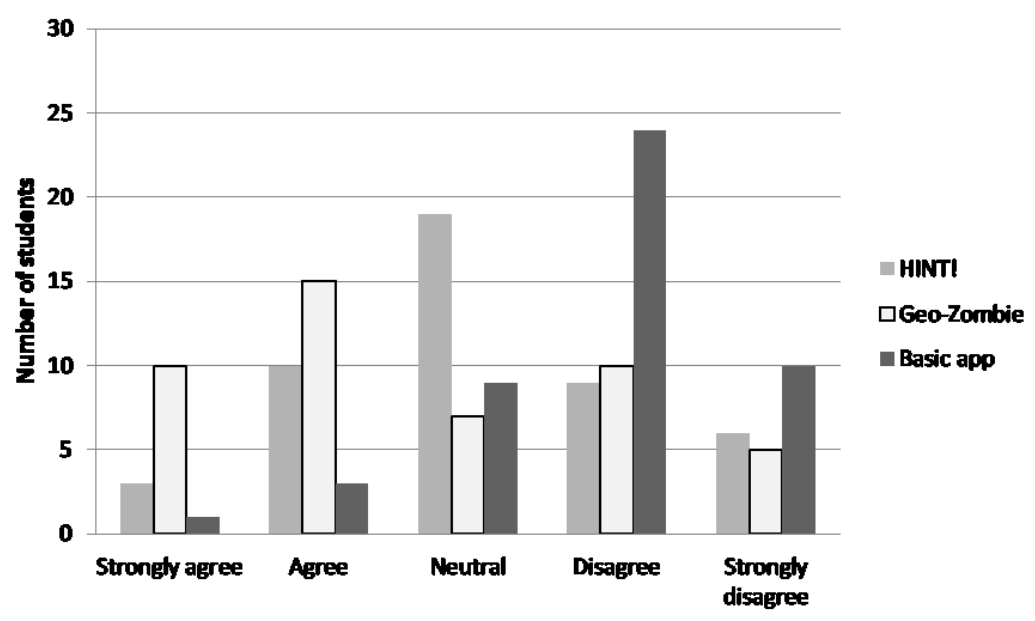

Fig. 11 Answers to the survey item "I come out from my daily paths"

In essence, with the use of Geo-Zombie, several participants broke the rigidity of their daily paths, urged by the need to escape from approaching zombies. Thus, in addition to exploring alternative walking paths, Geo-Zombie in some sense seems to have challenged the precise, algorithmic vision of those walkers who were used to follow always the same daily route.

A complete contrasting aptitude was expressed by Luca who was annoyed by the idea of being forced in changing daily routines:

"I founded the zombies idea very cool but I don't want to run away from zombies during my daily activities... I don't want run at all'" 
Another important aspect highlighted by the outcomes, is related to the awareness of the urban environment. As shown in Fig.12, Geo-Zombie and HINT! enforce the awareness of barriers/facilities in the urban environment, elements that are usually invisible in daily routine of avid walkers, and this is an important step in breaking boundaries of a (perceived) different community. Data show a correlation between the number of reports done and the awareness reached: (i) the basic app obtained 1.97 average reports and an awareness (both "agree" and "strongly agree" answers) increased of 25\% (12 of 48); HINT! got 6.60 average reports and an awareness increased of 51\% (24 of 47), and, finally, (iii) Geo-Zombie obtained 5.95 average reports and an awareness increased of 50\% (24 of 48). This emerged also from students' comments. For example, Maria emphasized:

"Using Geo-Zombie, I found a lot of unexpected urban elements that give problems to people with disabilities; just in the area which I supposed to know well!"”

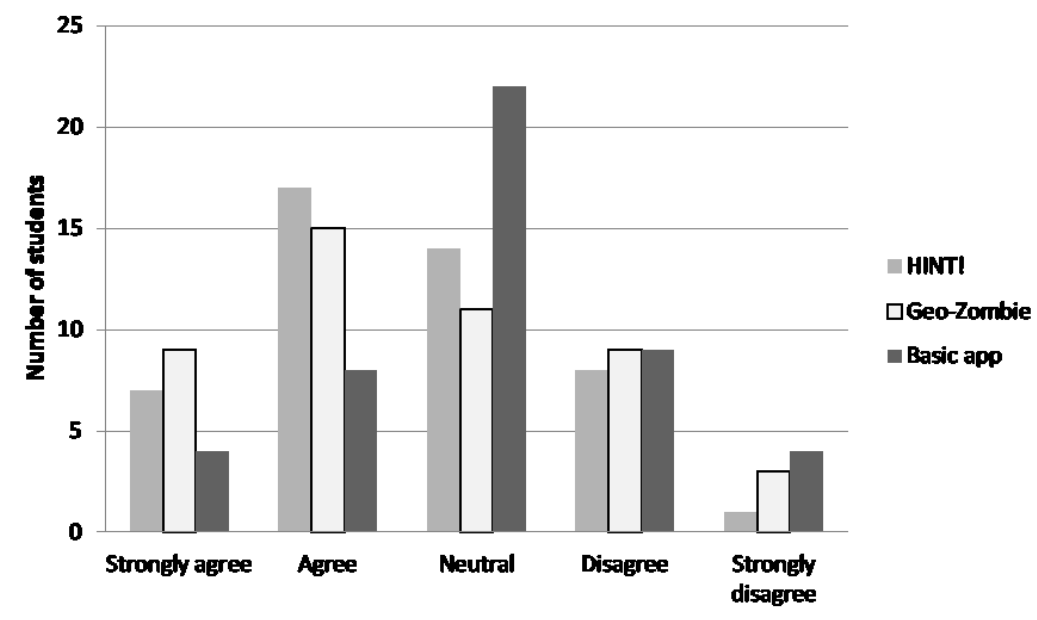

Fig. 12 Answers to the survey item "I'm more aware with the presence of barriers/facilities"

It is worth noting that for the Geo-Zombie app, the "strongly agree" answers are about $19 \%$ (9) while they are about 15\% (7) in HINT!, witnessing a higher level of engagement in the Geo-Zombie multimedia mobile app. In fact, some participants revealed that with Geo-Zombie they acquired an augmented amount of knowledge about the urban environment they used to live. According with Ilaria feeling:

"At a certain point, I was so worried about zombies that I started to walk quickly in the opposite direction to find news barriers! I really needed points to buy munitions... Zombies were so close to me!" 
This condition is the result of the game flow created by the pervasive game that allows to experience Geo-Zombie in a very immersive way, augmenting the focus on urban barriers/facilities. This outcome is also evident analyzing data in Fig. 13, related to the survey item "I tried to kill the largest number of zombies". The $47 \%$ of students (23) have been pushed by the game flow in killing as many zombies as possible. Anita affirmed:

"I gained a lot of munitions in my neighborhood just to walk versus the closer zombies and kill all of them! It was really funny!"

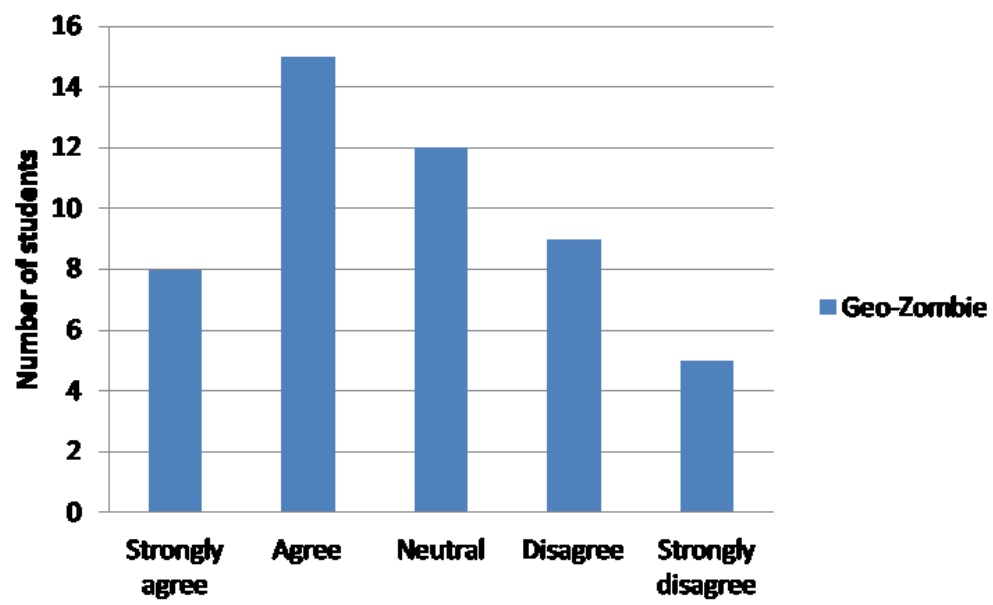

Fig. 13 Answers to the survey item "I tried to kill the largest number of zombies"

\section{Conclusion}

This paper described the design process, the implementation and field trials from which we went through with the aim of challenging the intuition of exploiting the zombie genre as an apotropaic medium in improving civic engagement. To do that we started from three basic design concepts that, after discussions, gave rise to five multimedia mobile apps, conceived to contrast exclusion through a GIS-enabled social multimedia tool. Focus group and experience prototyping were put to good use to select those multimedia mobile apps that, out of the initial five, better responded to our needs. At the end of this process, HINT! and Geo-Zombies emerged as the most suitable candidates for fostering civic engagement, being the former one based on an extrinsic motivation while the latter one resorted to an intrinsic motivation.

50 students were engaged in the final experimentation where the use of HINT! and Geo-Zombie were contrasted against a basic app (a simple app to map data without fun). Obtained data were analyzed under two points of view: quantitative and qualitative. In particular, qualitative results yield contrasting results. On the one hand, with Geo-Zombie, a 
number of students felt really engaged in playing, and consequently, in mapping urban accessibility. The intrinsic motivation of fighting zombie was so strong to push them out of their daily paths, breaking their algorithmic life and turning the everyday walking experience into an opportunity to augment their personal awareness of the surrounding environment. On the other hand, nonetheless, some people admitted to have experienced Geo-Zombie as deeply worrisome, due to the sense of impending disaster hanging over them on their usually relaxing walk. From the quantitative point of view, numerical data shows that Geo-Zombie is the multimedia mobile app that better transforms the mapping activity in an entertaining walk by exploiting GIS as a multimedia mean to encourage players in wandering and discovering new paths, collecting data about urban accessibility. This invites new reflections and discussions on the issue of urban data crowdsourcing while exploiting applications that provide fun or entertainment.

\section{Acknowledgement}

We thank all the participants (from the Madeira Interactive Technologies Institute and from the University of Bologna) involved in this study. We would also like to thank Steven Dow and Jodi Forlizzi (Carnegie Mellon University) who provided us with precious suggestions, feedbacks and ideas.

\section{References}

1. Bantinaki, K., 2012. The paradox of horror: fear as positive emotion. J. Aesthet. Art Crit., $70(4), 337-421$.

2. Bertram, D., 2015. Likert Scales are the meaning of life. URL: http://poincare.matf.bg.ac.rs/ kristina/topic-dane-likert.pdf (accessed June 20, 2015)

3. Buchenau, M., Suri, J.F., 2000. Experience Prototyping. In: Proceedings of the 3rd conference on Designing interactive systems: processes, practices, methods, and techniques, DIS '00. ACM, pp. 424-433.

4. Cabitza, F., Fogli, D., Piccinno, A., 2014. Fostering participation and co-evolution in sentient multimedia systems. Journal of Visual Languages and Computing 25(6), pp. 684694.

5. Carroll, N., 2003. The philosophy of horror: or, paradoxes of the heart. Taylor \& Francis.

6. Cordova, 2012. Discover plugins for your Apache Cordova project. URL: http://plugins.cordova.io (accessed May 8, 2015). 
7. Crimespotting, 2015. URL: http://stamen.com/projects/crimespotting (accessed April 16, 2015).

8. Csikszentmihalyi, M., 1991. Flow: the psychology of optimal experience. Harper Perennial.

9. Csikszentmihalyi, M., 2002. Flow: the psychology of happiness. Rider. New Ed edition.

10. Darabont, F., 2010. The walking dead, AMC TV channel.

11. Deci, E.L., Koestner, R. and Ryan, R.M., 1999. A meta-analytic review of experiments examining the effects of extrinsic rewards on intrinsic motivation. Psychological bulletin, $125(6)$, p.627.

12. De Certeau, M., 1988. The practice of everyday life. University of California Press.

13. Deterding, S., Dixon, D., 2011. Gamification: using game design elements in non-gaming contexts. In: Proceedings of the 2011 ACM SIGCHI Conference on Human Factors in Computing Systems, CHI '11. ACM, pp. 5-8.

14. Deterding, S., Björk, S.L., Nacke, L.E., Dixon, D., Lawley, E., 2013. Designing gamification: creating gameful and playful experiences. In: Proceedings of CHI '13 Extended Abstracts on Human Factors in Computing Systems, CHI EA '13. ACM, pp. 3263-3266.

15. Ding, C., Wald, M., Wills. G., 2014. A survey of open accessibility data. In: Proceedings of the 11th Web for All Conference, W4A '14. ACM, pp. 37:1-37:4.

16. Ferretti, S., Furini, M., Palazzi, C.E., Roccetti, M., Salomoni, P., 2010. WWW recycling for a better world. Commun. ACM., 53 (4), 139-143.

17. Fischer, G., 2010. End User Development and Meta-Design: Foundations for Cultures of Participation. Journal of Organizational and End User Computing 22(1), pp. 52-82..

18. Fischer, G., 2011. Understanding, fostering, and supporting cultures of participation. Interactions 18(3), pp. 42-53.

19. Google Maps, 2009. URL: https://maps.google.it (accessed April 16, 2015).

20. Haensch, A., 2013. When Fleeing Zombies (Or Flu), Cooperation Saves Lives. URL: http://www.npr.org/sections/health-shots/2013/07/30/207073336/when-fleeing-zombiesor-flu-cooperation-saves-lives (accessed April 16, 2015).

21. Hamari, J., Koivisto, J., Sarsa, H., 2014. Does gamification work? A literature review of empirical studies on gamification. In: Proceedings of the 47th Hawaii International Conference on System Sciences, HICSS. IEEE, pp. 3025-3034.

22. Hara, K., Azenkot, S., Campbell, M., Bennett, C.L., Le, V., Pannella, S., Moore, R., Minckler, K., Ng, R.H., Froehlich, J.E., 2013a. Improving public transit accessibility for 
blind riders by crowdsourcing bus stop landmark locations with google street view. In: Proceedings of the 15th International ACM SIGACCESS Conference on Computers and Accessibility, ASSETS '13. ACM, pp. 16:1-16:8.

23. Hara, K., Le, V., Froehlich, J., 2013b. Combining crowdsourcing and google street view to identify street-level accessibility problems. In: Proceedings of the 2013 ACM SIGCHI Conference on Human Factors in Computing Systems, CHI '13. ACM, pp. 631-640.

24. Hara, K., Sun, J., Moore, R., Jacobs, D., Froehlich, J., 2014. Tohme: detecting curb ramps in google street view using crowdsourcing, computer vision, and machine learning. In: Proceedings of the 27th annual ACM symposium on User interface software and technology, UIST '14. ACM, pp. 189-204.

25. Hung, S.Y., Durcikovab, A., Lai, H.M., Lin W.M., 2011. The influence of intrinsic and extrinsic motivation on individuals' knowledge sharing behavior. Int. J. Hum. Comput. Stud., 69 (6), 415-427.

26. Hunicke, R., LeBlanc, M., Zubek, R., 2004. MDA: A Formal Approach to Game Design and Game Research. Work. In: Proceedings of the Challenges in Games AI Workshop, Nineteenth National Conference of Artificial Intelligence. Press, pp. 1-5.

27. Kaiser, W.L. and Wood, D., 2001. Seeing through maps: The power of images to shape our world view. ODT Inc..

28. Keane, K., Nisi, V. Experience Prototyping: Gathering Rich Understandings to Guide Design. Emerging Research and Trends in Interactivity and the Human-Computer Interface, Hershey, $224-237$.

29. Jisun, 2015. Disability, Inclusion, and the Zombie Apocalypse. URL: http://kimchilatkes.com/2015/02/02/disability-inclusion-and-the-zombie-apocalypse (accessed April 16, 2015).

30. Latour, B., 1992. Where are the missing masses? The sociology of a few mundane artifacts. Shap. Technol. Soc. Stud. Sociotechnical Chang., 225-258.

31. Lefebvre, H., 2004. Rhythmanalysis: Space, Time and Everyday Life. Continuum.

32. Lehner, U., Baldauf, M., Eranti, V., Reitberger, W., Fröhlich, P., 2014. Civic engagement meets pervasive gaming. In: Proceedings of CHI '14 Extended Abstracts on Human Factors in Computing Systems, CHI EA '14. ACM, pp. 1483-1488.

33. Mirri, S., Prandi, C., Salomoni, P., 2014a. A context-aware system for personalized and accessible pedestrian paths. In: Proceedings of 2014 International Conference on High Performance Computing \& Simulation, HCPS. IEEE, pp. 833 - 840. 
34. Mirri, S., Prandi, C., Salomoni, P., Callegati, F., Campi, A., 2014b. On combining crowdsourcing, sensing and open data for an accessible smart city. In: Proceedings of the 2014 Eighth International Conference on Next Generation Mobile Apps, Services and Technologies, NGMAST. IEEE, pp. $294-299$.

35. OpenTripPlanner, 2015. URL: http://opentripplanner.usf.edu (accessed April 16, 2015).

36. Palazzi, C.E., Teodori, L., Roccetti, M., 2010a. Path 2.0: A participatory system for the generation of accessible routes. In: Proceedings of the 2010 IEEE International Conference on Multimedia and Expo, ICME. IEEE, pp. 1707-1711.

37. Palazzi, C.E., Roccetti, M., Marfia, G., 2010b. Realizing the unexploited potential of games on serious challenges. ACM Computers in Entertainment, 8, 4.

38. PhoneGap, 2015. Easily create apps using the web technologies you know and love: HTML, CSS, and JavaScript. URL: http://phonegap.com (accessed May 8, 2015).

39. Platts, T. K., 2013. Locating zombies in the sociology of popular culture. Sociology Compass, 7, 547-560

40. PostGis, 2015. URL: http://postgis.net (accessed October 16, 2015).

41. PostgreSQL, 2015. URL: http://www.postgresql.org (accessed October 16, 2015).

42. Prandi, C., Salomoni, P., Mirri, S., 2014. mPASS: integrating people sensing and crowdsourcing to map urban accessibility. In: Proceedings of the 2014 IEEE 11th Consumer Communications and Networking Conference, CCNC. IEEE, pp. $591-595$.

43. Prandi, C., Nisi, V., Salomoni, P., Nunes, N.J., 2015. From gamification to pervasive game in mapping urban accessibility. In: Proceeding of the 11th Biannual Conference on Italian SIGCHI Chapter, CHItaly 2015. ACM, pp. 126-129.

44. Quercia, D., Schifanella, R., Aiello, L.M., 2014. The Shortest Path to Happiness: Recommending Beautiful, Quiet, and Happy Routes in the City. In: Proceedings of the 25th ACM conference on Hypertext, HT '14. ACM, pp. 116-125.

45. Roccetti, M., Marfia, G., Palazzi, C.E., 2011. Entertainment beyond divertissment: using computer games for city road accessibility. Comput. Entertain. 9, 1-9.

46. Romero, G., 1968. Night of the living dead, New York, NY: The Weinstein Company, 2008, DVD.

47. Romero, G., 2010a. Who says zombies eat brains? URL: http://www.vanityfair.com/online/oscars/2010/05/george-romero (accessed April 16, 2015) 
48. Romero, G., 2010b. Questions for George Romero. URL: http://content.time.com/time/magazine/article/0,9171,1992390,00.html (accessed April $16,2015)$

49. Rosner, D.K., Roccetti, M., Marfia, G., 2014. The digitization of cultural practices. Commun. ACM, 57 (6), 82-87.

50. Rosner, D.K., Saegusa, H., Friedland, J., Chambliss, A., 2015. Walking by drawing. In: Proceedings of the 33rd Annual ACM Conference on Human Factors in Computing Systems, CHI '15. ACM, pp. 397-406.

51. Salomoni, P., Prandi C., Roccetti, M., Nisi, V., Nunes, N.J., 2015. Crowdsourcing Urban Accessibility: Some Preliminary Experiences with Results. In: Proceedings of the 11th Biannual Conference on Italian SIGCHI Chapter, CHItaly 2015.ACM, pp. 130-133.

52. Seaborn, K., Fels, D.I., 2015. Gamification in theory and action: A survey. Int. J. Hum. Comput. Stud., 74, 14-31.

53. Suchman, L., 1995. Representations of work: making work visible. Commun. ACM, 38 (9), 56-ff.

54. Sweetser, P., Wyeth, P., 2005. GameFlow: a model for evaluating player enjoyment in games. Comput. Entertain. 3, 1-24.

55. Von Ahn, L. and Dabbish, L., 2004, April. Labeling images with a computer game. In Proceedings of the SIGCHI conference on Human factors in computing systems, CHI '04 .ACM. pp. 319-326.

56. Von Ahn, L., Ginosar, S., Kedia, M., Liu, R., Blum, M., 2006. Improving accessibility of the web with a computer game. In: Proceedings of the SIGCHI Conference on Human Factors in Computing Systems, CHI '06. ACM, pp. 79-82.

57. WalkJogRun, 2015. URL: http://www.walkjogrun.net (accessed April 16, 2015).

58. Wheelmap, 2015. URL: http://wheelmap.org (accessed April 16, 2015).

59. Wiechman, B.M. and Gurland, S.T., 2009. What happens during the free-choice period? Evidence of a polarizing effect of extrinsic rewards on intrinsic motivation. Journal of research in Personality, 43(4), pp.716-719.

60. Wikitude, 2016. URL: http://www.wikitude.com/ (accessed March 1, 2016).

61. Wohlin, C., Runeson, P., Host, M., Ohlsson, M.C., Regnell, B. and Wesslén, A., 2000. Experimentation in software engineering: an introduction. 2000.

62. Wood, D., Fels, J., 1992. The Power of Maps. Guilford Publications. 